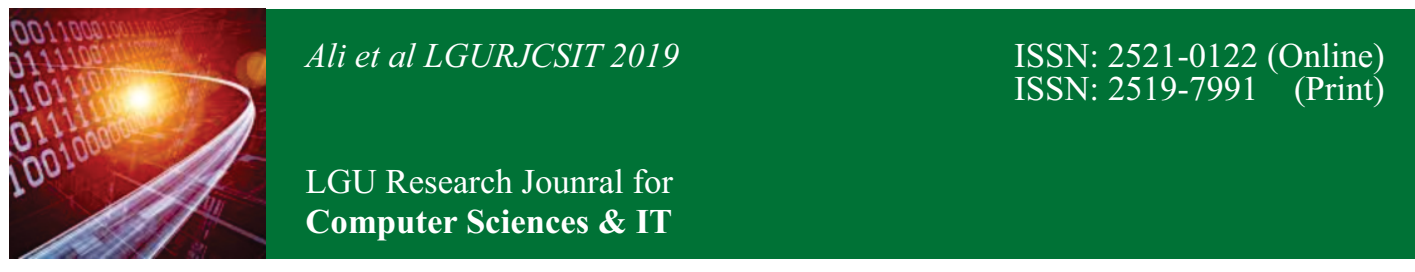

Vol. 3 Issue 4, October - December 2019

\title{
IoT Based Architecture for Basketball Supervision
}

Mubashir Ali, Sammya Hafeez, Mahnoor Khalid Paracha, Tehniyat Liaqat

${ }^{1 * 2,2,4}$ Department of Software Engineering, Lahore Garrison University, Lahore, Pakistan ${ }^{3}$ Department of Computer Science, Bahauddin Zakariya University, Multan, Pakistan 'mubashirali@lgu.edu.pk, 'dr.mubashirali1@gmail.com

\section{Abstract:}

Basketball is one of the most played games in the world with a huge amount of fan following and has a great number of basketballers. Sometimes players get severe lower body wounds such as ankle sprains, shortage of breath, head, teeth, hand, and fingers. Female players have a higher risk of knee injuries than male players. These are health issues that players face while playing basketball. Sports organizations spend millions to train fresh basketball players or for the development of the previous basketball players. The internet of things (IoT) made everyday things readable, controllable and recognizable through the internet and the wireless sensor networks. It is simply the network of interconnected devices that are embedded with sensors, software, and connectivity modules. Nowadays, with this growing technology it is possible to protect the life of players in the game as well as in training sessions, if we detect the problems early in players and appropriate actions will be taken to reduce adverse health effects which can be very dangerous. In this paper, we will propose an architecture for basketball based on the internet of things (IoT). The main goal behind this approach is to introduce a healthcare system based upon sensors, actuators, devices and telecommunication technologies to communicating real-time stats.

Keywords: Internet of things, Cloud computing, Edge Computing, Basketball Architecture, Healthcare, Wireless Body Area Networks, Sensors

\section{Introduction}

Basketball is a sport with 360 to 450 basketballers in the current National Basketball Association (NBA) and 144 to 180 basketballers in the Women National Basketball Association (WNBA). The Basketball Tournament (TBT) is mainly a singleelimination tournament played each summer in America and Canada, currently consists of 64 teams and usually broadcast by Entertainment and Supports Programming Network (ESPN). These tournaments are basically responsible for increasing the progress and taking care of the condition of the game around the world. Jonathan Mugar was the founder of TBT. NBA has also introduced an innovation that has been made progress in the game. They introduce a technology which is the Replay System in which alerts if a shot was discharged before the final buzzer or not. This system enables the couches to again view the footage of the game in good quality. This technology is implemented in 2002 in Western Conference finals. This system has made it very easy for the couches to make their last decision and it helps couches and players to see what they need to improve and where they made mistakes [1]. NBA is seeking ways to make Basketball a healthy sport too [2]. NBA players are known as the most paid players in the world by average annual salary per player. They also made an effort in the player's health issues. It has made it compulsory to add full-time mental health staff for the basketballers. The given system of IoT basketball will benefit the instructor so they should be able to take the authentic decision in terms of the medication of an injured player by informing the health staff on 
time [3].

The Internet of things is referred to as actuators, sensors and any kind of embedded systems and these systems have the capability to generate any kind of data globally in an automated manner [4]. Many applications can be developed through the possibilities offered by the internet of things, in many diverse scenes, such as healthcare and wellness, homes and industrial automation[5], automotive, smart grids, etc [6]. The new generation internet enables global communication with the world using advanced communication technologies like 4G, 5G. Wireless networks providing a base to the internet of things for effective communication. Among all enabling technologies of the internet of things, there are two such innovative systems that can be useful for development such as Radio Frequency Identification abbreviated as (RFID) and Wireless Sensor Network abbreviated as (WSN) presents two of the very most up and coming solutions. IoT providing mechanism to gather data from RFID or mobile devices, and sensors. RFID is a technology mainly used to record data, control the targets and it can also identify objects. RFID is an electronic chip-based technology or a system for identification. In this technology, any device can be controlled by radio waves [7]. Then it transfers the data to the first part and then to the second part. Moreover, telecommunication is generally sending or receiving the information by the wireless or nonwireless medium. The nodes of IOT must have the ability to communicate with another object such as machine and humans and this should be the machine to machine habitat. Internet of things can be enforced in many of the applications such as care of health [8], smart cities [9], agriculture [10], smart grid [11], smart building [12], home automation [5], saving energy, and many more. It enhances the lifestyle of individuals and society. A lot of development work has been done on the internet of things in the past. That work is reliant on the tools or methods, classifications, components and these are needed in a general place. ETSI's M2M is one of the most popular IoT standards. It has much recognition in terms of automated systems [11]. There are two parts to this standard. The first part consists of away, and its work is to collect important data from the devices of the system [11]. The other part is the connection part and executive, which consists of these factors such as storing data, accessing information securely, analyzing and routing [13].

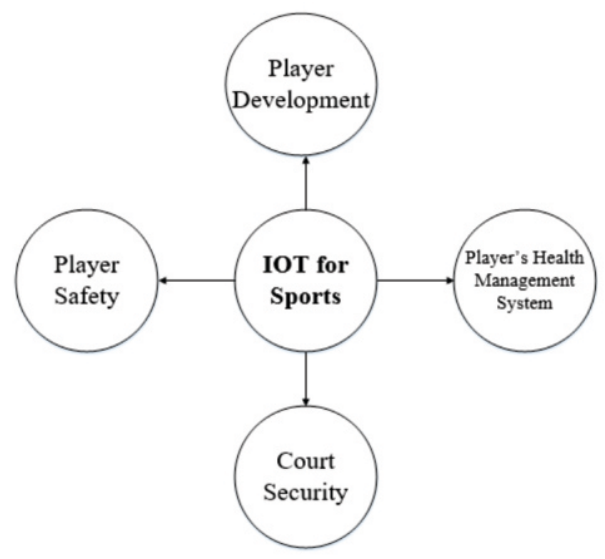

Figure. 1. IoT in Sports

Best technologies for examining human stats are Wireless Body Area Networks (WBANs) [14]. It can determine important physical functions such as heart rate, the motion of body and blood pressure [15]. Thus, it is possible to form a health system that keeps an eye on the health of a basketballer using automation which is based on the internet of things, and it is able to reduce the different health issues which can cause a negative effect on basketballers. In this new generation, the sensing devices enable us to connect to the networks by using interconnection technology [16]. Furthermore, IPv6 is placed by 6LoPWAN (Low power wide area network) on comparatively small devices so the data is transferred with strong packet transportation and low power utilization. We will introduce IoT Basketball, and its purpose is to attentively observe basketballers when they are playing, and if unusually any injury or accident occurs the problems can be recognized and solved on time [17]. It basically works by putting heat gadgets on the basketballers when they are playing in the tournaments or in trails to instantiate essential frameworks mainly temperature of the body, and rates which include sweat, heart, body, and respiration [18].

In section 2 we will talk about the technology used in this application and it includes the factors which are required for this architecture. In the 3rd section, we will discuss a case study with two cases that highlight the importance of this architecture and finally propose IOT based architecture with four layers perspective. 4th section is all about the 
illustration of some difficulties that could be encounter while implementing this approach in practice. Lastly, in the 5th section, we conclude our work and provide guidelines for further improvements.

\section{LITERATURE REVIEW}

Nowadays, technology has become the most important part of people's life. It has made life so much easier, safe, reliable and convenient by providing an enormous amount of services. Wearable monitoring devices play a major role and are very helpful in monitoring and collecting physiological data such as blood rate, daily activity and oxygen level which improves the quality of life. In the future, the health care system will be very improved with the use of these devices because these can detect disease early on and prevent it from occurring by providing proactive wellness management. The most important technology which is developed recently and also implemented in the health care system is Wireless Body Area Networks [18]. Most of the diseases occur because there is no time-based checkup for the players in order to get quick treatment by reporting it in the first place. Wireless Body Area Networks (WBANs) help the players to get the treatment accordingly and they don't have to stay in hospitals for a long time. It consists of actuators and small biomedical sensors that are found on the patient's body. Sensors measure the body parameters and actuators work on the data received by sensors. This technology allows monitoring the temperature of the body, heartbeat and blood pressure of the player. With the help of Internet Communication Center, this data is sent to electronic devices such as phones, laptops, and tablets. Data is gathered by the sensors [19]. WBANs provide the possible solutions and are pro-active, very fast and comparatively affordable as compared to the other health monitoring systems.

WBANs are the core technology that is used to report any abnormality of the human body and to diagnose any kind of disease [20]. After analyzing the data, it also tells the required solution or treatment of the problem. Moreover, WBANs are non-invasive technology and economical solutions for any healthcare application. Other wireless devices such as WSNs, WPAN, MiWi, ZigBee, WLAN along with the internetwork with the WBANs.
WPAN and WLAN abbreviated as Wireless Personal Area Networks and Wireless Local Area Networks. WBANs are an emerging technology with the aim to provide a comfortable life by providing suitable care systems for its patients. Many health care systems are developed on the basis of WBANs and research efforts are also in progress to enhance this technology. There are other wireless networks too which are exploring in the health sector such as Vimax and wireless body sensor networks. The main objective of this paper is to develop a system for health care which's purpose is to report the player's disease early so the players can not suffer from it. There is a lack of attention towards the health care system for the players in the sport that is why we are proposing this Basketball Architecture based on IoT. However, many research articles proposed on this by other models. [21] Presents a monitoring system that can be used daily and also in the field of sports. This system consists of body nets and totally dependent on body contact. It can measure many body diseases such as the amount of oxygen, heartbeat, sweat rate and blood pressure which can be found by photoplethysmography signal. When data is received, it gives solutions to the people who are in the field of sport as well as to the people who do daily physical exercise. Another article on the health care system is proposed by [22] in which a personal computer application software is implemented and is based on WBANs. These computers work on the embedded sensors which are used to monitor body movements and ECG sensors to see heart activities. These two systems worked so well but there is one limitation that is they can only work in a specific environment. And develop a system that is convenient for the players while playing and training. Upcoming researches have shown interest in developing a system for the players which is light-weight and cannot irritate the player while playing such as bracelets, headbands, shorts, and T-shirts. With the emerging technology people prefer WBANs for health care monitoring as it is more sufficient and reliable. [23] introduced a device that is a shoe-based wearable device and its purpose is to measure different postures of the human body such as standing, cycling, jumping, walking and running, taking steps in ascending or descending manner and helps the people suffering from obesity. Furthermore, a system, [24] was proposed which works with $\mathrm{pH}$ monitoring 
systems having $\mathrm{pH}$ sensors and it measures the amount of sweat of the patient in order to see the problem. [25] Introduced wearable shirts. These shirts measure the player's ECG signals and its activities in order to detect any unusual activity in the player's body.

As many research papers are focusing on developing a system on health care in sports and many of them are already introduced in different terms by using different technologies. [26] Introduced a system using IOT technology for patients in which they can stay at home such as an easy environment for them. It contains three parts mainly sensors, Microsoft XBOX and ICU monitoring system. The writers of [27] introduced an architecture based on IOT to build a system based on health care using ETSI's M2M standards. Bluetooth has been also introduced as WPAN protocol and is very famous for its large connection availability for current medical systems. Yet there is a shortage of research on introducing or making a system for the sports in order to give facilities for monitoring which is based on the architecture of IoT. IoT Basketball provides a system that prevents the players from any kind of incident, sports-related risks, and major injuries. Basket baller's activity can be seen with this system by measuring different parameters and also measuring the parameters of the area such as intensity and temperature of that place. This work can be done by using different technologies and sensing devices which can be placed on the player's kit or by installing it under the player's skin or in his tissues. This approach enables the communication with Constrained Application Protocol and IP address. These technologies can be accessed by the web services. RFID is implemented in the system for the basket baller's identification and the Routing Protocol for Low Power (RPL) is implemented in IOT basketball.

\section{PROPOSED ARCHITECTURE}

\subsection{Case Study}

As we already know basketball is one of the most played games in the world with 2 million fans and 450 plus professional basketball players from all around the world. It is played in the rectangular court and you scored the points depending on which side you throw the ball into the hoop. The ball can be moved in the court by plopping and passing the ball to the other players. The end result of the game is declared by the referees and the team with the most points will win the game. The teams will consist of 12 players and only 5 are allowed to play in the court. There are different positions by the players such as center, defensive forward, offensive forward, defensive guard and point guard. Each player then will take his positions but also allowed to move in the court as per requirements [28]. A case study is being illustrated in this section that how IoT Basketball will help the basketballers in terms of their health issues which occurs during the game or training such as accidents or sudden illness. We will briefly talk about the problems and see their solutions. Figure 2 shows the basketball court with dimensions. Proposed IoT based architecture for basketball which describes the technologies that will be enforced to provide the players with safe and effective outcomes which leads them to the higher levels of performance and quick recovery which ensures the success of players, while making sure the players are healthy and fit as they were before.

Taking in view the case study of a first player. First Player's name is Charlie Puth. Charlie has been in this game for more than nine years and is treated as the professional in the game. In his game history, there is no clue of any major illnesses in his medical background. While he was playing in the field one day, he became unconscious. There can always a risk with all the players that other than unconsciousness they can face any health issue on the field while playing the game. It includes heart attack, ankle sprains, fatigue, eye to eye happenings and many other small injuries. Our system will help the coaches and supervisors to monitor the health issues of players before the game and even in the field without any delay because our technology operates immediately.

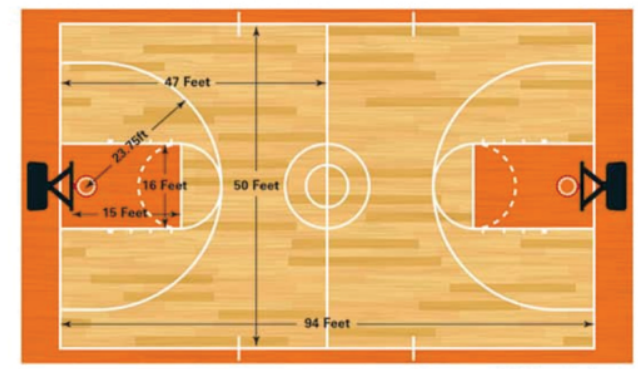

Figure. 2. Basketball Court 
Now talking about the case study of another player. The second Player's name is Will Smith. Smith has been in this field for almost five years and he has never been sick on the field. One day suddenly, he suffered from a minor heart attack. This happened because there was no system to measure the health of the players in the field or before the game. In the next section, we will illustrate how this technology will help in preventing the situations and how it responds if the player faces any major health issue.

Firstly, [29] we used RFID technology to get personal information about the player, which includes his good name, registration number, designation, his medical background and he plays with which team. The environment of the court along with the player's condition will be determined. These include mainly the temperature of the court, the intensity of light, body temperature, heartbeat, level of oxygen, sweat rate, blood pressure, and respiration rate. We will use the default gateway to transform the information or the signals to the Adafruit cloud. Adafruit cloud has many built-in functions and libraries which are available for health care monitoring. We are using the MiWi protocol for this instance. After Adafruit cloud analyzed the information, a notification is sent to the coach or the team supervisor as a warning by an electronic device that tells them that Charlie in first case and smith in the second case is not okay, and they need medical assistance immediately and take them out of the court. They are provided with medical help. If after the recovery something happened to any of them the technology will be operated in the same way. Our system will make sure that the players will be alright and they are doing well and we do this by monitoring them. This system will get to know the condition of the player even before himself and how to fix or prevent it from happening. It means that there will be a minimum possibility of any injury or major health issue while he was focusing on his game.

\subsection{IOT Basketball Architecture}

IoT allows different devices to communicate with each other via the internet or wireless networks. To support the case studies we mentioned above we also proposed an IoT architecture which is shown in figure 3. We used different sensors that are lightweight, got their own IPv6 capabilities (6oLWPAN) and have low power consumption. It measures the physical parameters such as sweat rate, heartbeat, motion, and temperature of the body, breathing rate, and blood pressure. This information is sent to the cloud through wireless sensor networks with the gateway. To measure the environment of the court we use other sensors too which includes the temperature and humidity in the court. This layer in the system is known as the perception layer. To communicate with the default gateway the Constraint Application Protocol (CoAP) is used in the sensors [30].

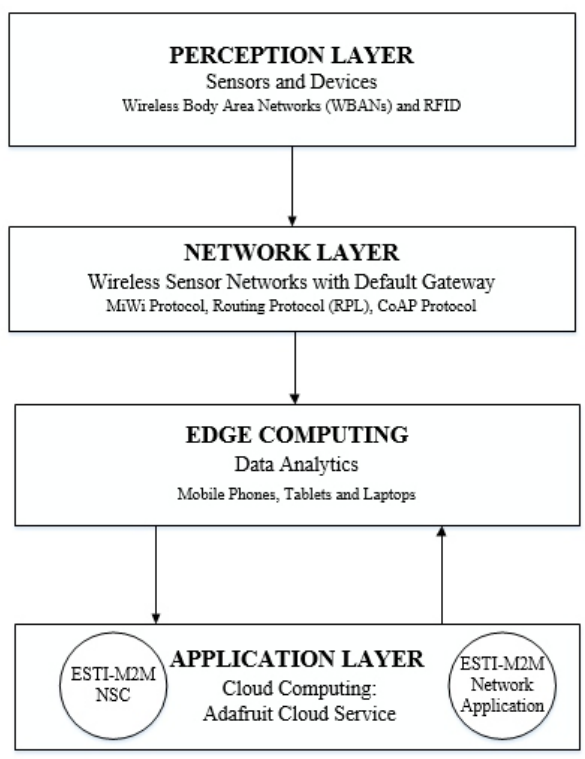

Figure. 3. IOT Basketball Architecture

This protocol is used to avoid conjunction between the technologies and it makes a gateway to integrate with the web by using HTTP. Furthermore, CoAP has many advantages such as multicast support, low header overhead, asynchronous message exchange and it is based on the protocol which is Datagram Protocol (UDP) which is used to transport with an application layer.

The second block in the figure referred to as a Network layer that collects all the data from the perception layer and transfers over the cloud. MiWi protocol is the default communicator which is used in the IoT basketball architecture. Data collected from the perception layer is transferred over the cloud using the MiWi base station. The ETSI's M2M is divided into 2 parts. ETSI's M2M NSC is used for the network application. Network application works for the registration, security, routing, and NAT. MLA interface is a bidirectional data flow is provided 
by MLA Interface and it works as a passage between the network application and NSC. To perform IOT Basketball in the world there are many functions and characteristics that a system must operate. The next section provides the details used in every part. The basketball IoT architecture consists of four layers that are under:

\section{Perception Layer \\ 2. Network Layer \\ 3. Edge Computing \\ 4. Application Layer}

\section{A. Perception Layer in IoT Basketball Architecture}

This layer consists of sensors having two networks. We use the wireless body area networks (WBANs) to measure the health condition of the basketballer. These kinds of sensors must be lightweight because players need to wear them such as shirts, Bracelets, and shorts. To communicate with the gateway of the system, every sensor must have its own IPv6 (6LoWPAN) and must be working with Routing Protocol for Low-Power (RPL). The CoAP Protocol works with the sensors to send and receive the information. Moreover, the devices must fulfill the security capabilities and QoS requirements. The devices around the court which measure the temperature and humidity do not have the same capabilities as the body sensors, but they need to cooperate with the body sensors in order to transmit data over the cloud computing platform known as adafruit cloud.

\section{B. Network Layer in IoT Basketball Architecture}

In this system, the MiWi protocol is used for the base station. The base station must be connected with a network domain with a wireless connection. The base station also needs to work with the 6LoWPAN, which means supporting the IEEE 802.11 standard of wireless communication. Moreover, the default gateway must implement the CoAP Protocol to send and receive the information from the sensors. It enables QoS Requirements and security efficiency [31].

\section{Edge Computing Layer in IoT Basketball Architecture}

Edge computing refers to end node based infrastructure that processes instant data in realtime fashion for quick response [32]. In layer three, the sensed data is processed and notified to supervisors before sending it to overcloud. The complex operations are performed overcloud. It also performs data-handling activities or other network operations. It performs the data analysis instantly and presents the results to the users. It delivers the data to the endpoint devices such as mobile phones, laptops, and tablets. It performs computation on the edge devices. Moreover, these devices move large amounts of processed data it improves the latency, quality of service, reliable and quick response and cost of transmission.

\section{Application Layer in IoT Basketball Architecture}

The Cloud computing service known as the Adafruit cloud is used over the application layer [33]. It examines traffic in the data that receives from the technologies when the conjunction occurs in order to facilitate the coach or the superintendent of the game. To analyze the injuries and health conditions of the basketballers we can possibly use predictive analytics in data mining. Adafruit cloud services ensure the quality of service and security efficiencies. The information of the players should be stored in a secure place. Furthermore, the Adafruit cloud is more suitable as compared to the other software and used to provide the correct information to the superintendent of the game. It should be able to give complete information about the basketballer such as health conditions, preceding ailments, and weaknesses.

\section{DISCUSSION}

We can several hurdles while implementing this system for basketball which is as follows:

- $\quad$ Some players don't like the idea of their private information being gathered by the sensing devices because their privacy is more important and if it got leaked to the other people it can cause damage and have an adverse effect on their life. 
- Any communication system will need to be upgraded as the passage of time, technology will be evolving in performance levels, the quality of gadgets, speed of processing and many more. For this, we should be updated about the new happenings.

- In order to implement the required technologies in the court and the gadgets, this whole process can be very expensive as it changes the whole structure of the court.

- There can be technological errors such as the coach does not receive instant notifications edge computing layer and the connection between the sensors and the default gateway.

- $\quad$ To manage all the gadgets, automation and structure of the system to the high level.

- The ability of computer systems and software to work perfectly is crucial to effectiveness. So they have to work with flexibility in order to function properly and to provide accurate results.

- $\quad$ IoT basketball consists of different sensors and layers in order to work effectively between the basket baller's wearable devices in the body and to the superintendent electronic medium. It makes an effective interaction between both.

- Many architectures of IoT have been presented before to find a relevant standard to form an IoT Basketball system and it is essential for us to find one above of the success.

\section{CONCLUSION}

IoT refers to interconnected communication between actuators, sensing devices and embedded systems. It strengthens the style of living in a society via effective automation. It has much utilization which consists of smart cities, health care, smart buildings, home automation, intelligent traffic system and many more. In this research, we have proposed a basketball architecture, which aims to monitor basketballers while they are playing or in the trainings so if any injury or accidents happened in the game it can be overcome and given the solutions. This can be made possible by putting the wearable sensing devices on basketballers during the game and transmits all the information to the application layer for storing and processing of the data, and then finally sends information to the superintendent with the help of electronic devices such as phones, laptops and tablets so they can see if anything happened to the player in the court or not. The architecture of this system uses ETSI's $\mathrm{M} 2 \mathrm{M}$ standard. In the future, we will enhance this architecture for other sports such as football, volleyball to prevent health issues. TinyOS simulation will be used for the implementation of this system and to address any kind of circumstance that can occur.

\section{CONFLICT OF INTEREST}

On behalf of all authors, the corresponding author states that there is no conflict of interest.

\section{Acknowledgment}

We would like to thank journal editor, area editor and anonymous reviewers for their valuable comments and suggestions to help and improve our research paper.

\section{REFERENCES}

[1] B. Chettri, S. Mishra, B. L. Sturm, and E. Benetos, "Analysing the Predictions of a CNNBased Replay Spoofing Detection System," in 2018 IEEE Spoken Language Technology Workshop, SLT 2018 - Proceedings, 2019.

[2] C. E. Gross, J. Huh, and J. A. Nunley, "Basketball," in Foot and Ankle Sports Orthopaedics, 2017.

[3] M. A. Ikram, M. D. Alshehri, and F. K. Hussain, "Architecture of an IoT-based system for football supervision (IoT Football)," in IEEE World Forum on Internet of Things, WF-IoT 2015 - Proceedings, 2015.

[4] T. Jensen and M. Durham, "Internet of things," Advancing Microelectronics, 2017.

[5] M. Alaa, A. A. Zaidan, B. B. Zaidan, M. Talal, and M. L. M. Kiah, "A review of smart home applications based on Internet of Things," Journal of Network and Computer Applications. 2017.

[6] J. Lin, W. Yu, N. Zhang, X. Yang, H. Zhang, and W. Zhao, "A Survey on Internet of Things: Architecture, Enabling Technologies, Security and Privacy, and Applications," IEEE Internet Things J., 2017. 
[7] S. Amendola, R. Lodato, S. Manzari, C. Occhiuzzi, and G. Marrocco, "RFID technology for IoT-based personal healthcare in smart spaces,”IEEE Internet Things J., 2014.

[8] G. Kaur, P. Tomar, and P. Singh, Internet of Things and Big Data Analytics Toward NextGeneration Intelligence. 2018.

[9] Z. Kamal, A. Mohammed, E. Sayed, and A. Ahmed, "Internet of Things Applications , Challenges and Related Future Technologies," World Sci. News, 2017.

[10] A. K. Saha et al., "IOT-based drone for improvement of crop quality in agricultural field," 2018 IEEE 8th Annu. Comput. Commun. Work. Conf. CCWC 2018, vol. 2018-Janua, pp. 612-615, 2018.

[11] L.-Y. Chen and C.-Y. Huang, "An open internet of thing architecture integrating OnEM2M and OGC sensorthings API standards," in Proceedings - 39th Asian Conference on Remote Sensing: Remote Sensing Enabling Prosperity, ACRS 2018, 2018.

[12] I. Rosadi and S. P. Sakti, "Low-cost wireless sensor network for small area in a building," in Proceedings - 2017 International Seminar on Sensor, Instrumentation, Measurement and Metrology: Innovation for the Advancement and Competitiveness of the Nation, ISSIMM 2017, 2017.

[13] G. Katsikogiannis, D. Kallergis, Z. Garofalaki, S. Mitropoulos, and C. Douligeris, "A policy-aware Service Oriented Architecture for secure machine-to-machine communications," Ad Hoc Networks, 2018.

[14] K. C. Karthika, "Wireless mesh network: A survey," in Proceedings of the 2016 IEEE International Conference on Wireless Communications, Signal Processing and Networking, WiSPNET 2016, 2016.

[15] . K. L . K., "B O D Y A R E A NETWORKS,” Int. J. Res. Eng. Technol., 2016.

[16] "Evaluation of Wireless Body Area Networks," Int. J. Innov. Technol. Explor. Eng., 2019.
[17] S. Al-Janabi, I. Al-Shourbaji, M. Shojafar, and S. Shamshirband, "Survey of main challenges (security and privacy) in wireless body area networks for healthcare applications," Egyptian Informatics Journal. 2017.

[18] M. Ghamari, B. Janko, R. S. Sherratt, W. Harwin, R. Piechockic, and C. Soltanpur, "A survey on wireless body area networks for ehealthcare systems in residential environments," Sensors (Switzerland), 2016.

[19] S. Majumder, T. Mondal, and M. J. Deen, "Wearable sensors for remote health monitoring,"Sensors (Switzerland). 2017.

[20] I. Lourenço and J. Costa, "In-shoe sensor system with an embedded user interface and wearable leg unit," in 6th IEEE Portuguese Meeting on Bioengineering, ENBENG 2019 Proceedings, 2019.

[21] Y. Fu and J. Liu, "Monitoring system for sports activities using body area networks," in BODYNETS 2013 - 8th International Conference on Body Area Networks, 2013.

[22] C. Otto, A. Milenković, C. Sanders, and E. Jovanov, "System architecture of a wireless body area sensor network for ubiquitous health monitoring," J. Mob. Multimed., 2006.

[23] E. S. Sazonov, G. Fulk, J. Hill, Y. Schutz, and R. Browning, "Monitoring of posture allocations and activities by a shoe-based wearable sensor," IEEE Trans. Biomed. Eng., 2011.

[24] D. Morris et al., "Wearable sensors for monitoring sports performance and training," in Proc. 5th Int. Workshop on Wearable and Implantable Body Sensor Networks, BSN2008, in conjunction with the 5th Int. Summer School and Symp. on Medical Devices and Biosensors, ISSS-MDBS 2008, 2008.

[25] Y. D. Lee and W. Y. Chung, "Wireless sensor network based wearable smart shirt for ubiquitous health and activity monitoring," Sensors Actuators, B Chem., 2009.

[26] I. Chiuchisan, H. N. Costin, and O. Geman, "Adopting the internet of things technologies in health care systems," in EPE 2014 - Proceedings of the 2014 International 
Conference and Exposition on Electrical and Power Engineering, 2014.

[27] P. Diogo, L. P. Reis, and N. V. Lopes, "Internet of Things: A system's architecture proposal," in Iberian Conference on Information Systems and Technologies, CISTI, 2014.

[28] B. Alamar, "Basketball on Paper: Rules and Tools for Performance Analysis," J. Sport Manag., 2016.

[29] Kubo, "The research of IoT based on RFID technology," in Proceedings - 7th International Conference on Intelligent Computation Technology and Automation, ICICTA2014, 2015.

[30] I. J. Shin, D. S. Eom, and B. K. Song, "The CoAP-based M2M gateway for distribution automation system using DNP3.0 in smart grid environment," in 2015 IEEE International Conference on Smart Grid Communications, SmartGridComm 2015, 2016.

[31] M.A. Razzaque, M. T. Hira, and M. Dira, "QoS in body area networks: A survey," ACM Transactions on Sensor Networks. 2017.

[32] W. Yu et al., "A Survey on the Edge Computing for the Internet of Things," IEEE Access. 2017.

[33] R. Gayathri and S. K. Vasudevan, "Internet of things based smart health monitoring of industrial standard motors," Indones. J. Electr. Eng. Informatics, 2018. 